\title{
Constraining the ages of the fireballs in the wake of the dlrr galaxy VCC 1217/IC 3418
}

\author{
Mattia Fumagalli ${ }^{1,2}$, G. Gavazzi ${ }^{2}$, R. Scaramella ${ }^{3}$, and P. Franzetti ${ }^{4}$ \\ ${ }^{1}$ Leiden Observatory, Leiden University, PO Box 9513, 2300 RA Leiden, The Netherlands \\ e-mail: fumagalli@strw.leidenuniv.nl \\ 2 Dipartimento di Fisica G. Occhialini, Università di Milano-Bicocca, Piazza della Scienza 3, 20126 Milano, Italy \\ e-mail: giuseppe.gavazzi@mib.infn.it \\ 3 INAF - Osservatorio Astronomico di Roma, via Frascati 33, 0040 Monte Porzio Catone (RM), Italy \\ e-mail: roberto.scaramella@oa-roma.inaf.it \\ 4 INAF, IASF-Milano, via Bassini 15, 20133 Milano, Italy \\ e-mail: paolo@lambrate.inaf.it
}

Received 23 July 2010 / Accepted 5 November 2010

\section{ABSTRACT}

\begin{abstract}
Context. A complex of $\mathrm{H} \alpha$ emitting blobs with strong FUV excess is associated to the dIrr galaxy VCC 1217/IC 3418 (Hester et al. 2010, ApJ, 716, L14), and extends up to $17 \mathrm{Kpc}$ in the southeast direction. These outstanding features can be morphologically divided into diffuse filaments and compact knots, where most of the star formation activity traced by $\mathrm{H} \alpha$ takes place.

Aims. We investigate the properties of the galaxy and the blobs using a multiwavelength approach to constrain their origin.

Methods. We collected publicly available data in UV and $\mathrm{H} \alpha$ and observed the scene in the optical $U, g, r, i$ bands with LBT. The photometric data allows evaluation of the star formation rate and performance of a SED fitting separately for the galaxy and the blobs in order to constrain their stellar population age. Moreover we analyzed the color and luminosity profile of the galaxy and its spectrum to investigate its recent interaction with the Virgo cluster.

Results. Our analysis confirms that the most plausible mechanism for the formation of the blobs is ram pressure stripping by the Virgo cluster IGM. The galaxy colors, luminosity profile and SED are consistent with a sudden gas depletion in the last few hundred Myr. The SED fitting of the blobs constrains their ages in $<400$ Myr.
\end{abstract}

Key words. galaxies: clusters: individual: VCC1217/IC3418 - galaxies: evolution - galaxies: irregular

\section{Introduction}

Recent studies of the Virgo cluster (Boselli et al. 2008), Coma supercluster (Gavazzi et al. 2010), Perseus cluster (Penny \& Conselice 2010) and Shapley supercluster (Haines et al. 2006) invoke ram pressure stripping (Gunn \& Gott 1972) as the responsible process for a significant migration of galaxies from the blue cloud to the red sequence, via suppression of the star formation by gas ablation of low-mass galaxies in regions of high galactic density. The necessary ingredient of these "near-field cosmology" approaches is that significant infall of low-mass starforming objects exists along the filamentary structures onto the densest clusters. These galaxies have their star formation truncated on a short timescale from the interaction with the IGM.

Observations of stripped gas are frequent in the local Universe. Long narrow $\mathrm{H} \alpha$ tails, stretching up to $150 \mathrm{Kpc}$, are reported in the Virgo cluster (Kenney \& Koopmann 1999), Abell 1367 (Gavazzi et al. 2001; Cortese et al. 2006), and Coma cluster (Yagi et al. 2010) associated to infalling galaxies. There is, however, little evidence that star formation ignites in the stripped wakes, except in a few cases. Cortese et al. (2007) discovers for the first time a complex of star-forming blobs in the trails of two spiral galaxies belonging to two clusters at $z=0.2$, and Yoshida et al. (2008) finds a unusual complex of blue "fireballs" associated to the Coma galaxy RB 199. Smith et al. (2010) identifies 13 objects in the Coma cluster with asymmetric ultraviolet morphologies, finding that the majority of the tails are pointing away from the cluster center. These cases of star formation in the wakes of stripped galaxies are remarkably similar to the hydrodynamical simulations by Kapferer et al. (2009).

Recently Hester et al. (2010) has reported the discovery of a similar object in the Virgo cluster, associated to the dIrr galaxy VCC 1217/IC 3418. We have been independently studying the same system with deep LBT photometry in addition to public $\mathrm{H} \alpha$ and GALEX-UV data, to constrain the ages of the galaxy and the fireballs via SED fitting.

Throughout this paper we assume a standard cosmology and a distance module of $31 \mathrm{mag}$ for the Virgo cluster A corresponding to a distance of $17 \mathrm{Mpc}$, as in Gavazzi et al. (1999).

\section{The data}

VCC 1217 was observed by GALEX in March 2004 in the Near UltraViolet (NUV, 1750-2750 ̊) and in the Far UltraViolet (FUV, $1350-1750 \AA$ ) bands with exposure times of $\approx 4000$ and $\approx 1600$ s respectively ${ }^{1}$. A narrow $\mathrm{H} \alpha$ band image was taken at the ESO $3.6 \mathrm{~m}$ telescope in 2004 (see Gavazzi et al. 2006). Sources with an $\mathrm{H} \alpha$ surface brightness higher than $\sigma_{\min }=$ $3.16 \times 10^{-17} \mathrm{erg} / \mathrm{s} / \mathrm{cm}^{2} / \operatorname{arcsec}^{2}$ were detected $(2 \sigma$ of the background). The galaxy is undetected at $21 \mathrm{~cm}$, as reported by Hoffman et al. (1989), who used the Arecibo telescope to put

\footnotetext{
${ }^{1}$ Significantly shorter than quoted by Hester et al. (2010) who used additional GALEX observations that are not yet public.
} 
Table 1. Observation log.

\begin{tabular}{|c|c|c|c|c|}
\hline Instrument & Filter & Seeing & Date (yy/mm/dd) & Exposure time \\
\hline LBT & $U$-spec & $1.80 \operatorname{arcsec}$ & $\begin{array}{l}2008-02-01,02 \\
2008-04-03,04\end{array}$ & $25 \times 240 \mathrm{~s}$ \\
\hline LBT & $g$-SDSS & $1.35 \operatorname{arcsec}$ & $\begin{array}{l}2008-04-03,04 \\
2009-02-22 \\
2009-05-28\end{array}$ & $28 \times 150 \mathrm{~s}$ \\
\hline LBT & $r$-SDSS & $1.47 \operatorname{arcsec}$ & $\begin{array}{l}2008-02-02 \\
2008-04-03,04 \\
2009-05-28\end{array}$ & $32 \times 150 \mathrm{~s}$ \\
\hline LBT & $i$-SDSS & $1.29 \operatorname{arcsec}$ & $\begin{array}{l}2009-02-01,02 \\
2008-04-03,04\end{array}$ & $34 \times 240 \mathrm{~s}$ \\
\hline ESO 3.6 & $r$ Gunn & $1.13 \operatorname{arcsec}$ & 2005-04-21 & $240 \mathrm{~s}$ \\
\hline ESO 3.6 & 692 & $1.13 \operatorname{arcsec}$ & 2005-04-21 & $1800 \mathrm{~s}$ \\
\hline GALEX & $N U V$ & $4.95 \operatorname{arcsec}$ & 2004-03-11 & $1597+1161+1690 s$ \\
\hline GALEX & $F U V$ & $4.05 \operatorname{arcsec}$ & 2004-03-11 & $1597 \mathrm{~s}$ \\
\hline ESO 3.6 & EFOSC spectrometer & & $2002-03-17$ & $2400 \mathrm{~s}$ \\
\hline
\end{tabular}

a stringent upper limit of $M_{\odot} \approx 3.46 \times 10^{6}$ on the HI gas in the galaxy. Optical observations were obtained at the LBT on different nights in the first semester of 2008, using the prime focus LBC cameras (http: //lbc .mporzio.astro.it/) with 4 filters: $U_{\text {spec }}$ and $g, r, i$ in the SDSS system. The seeing ranged between 1.4 and 2 arcsec. Data was reduced using the LBC standard pipeline by the LBC support team, and final images were typically composed of stacks of $<20$ dithers.

The photometric calibration was performed by crosscorrelating the flux of 27 stars in the field with those in the SDSS, resulting in zero points with an error of less than 0.02 mag. A summary of observations is found in Table 1. Magnitudes are given in the $\mathrm{AB}$ system throughout the paper.

\section{The galaxy}

\subsection{Morphology and photometry}

VCC 1217 is a dIrr (Nilson et al. 1973) low-surface brightness galaxy located near the center of the Virgo cluster, approximately 1 degree south of M 87 ( $0.31 \mathrm{Mpc}$ projected distance). It has a redshift of $38 \mathrm{~km} \mathrm{~s}^{-1}, \sim 1000 \mathrm{~km} \mathrm{~s}^{-1}$ lower than the mean redshift of Virgo cluster A. At this location the emission from the hot IGM is at its peak intensity (Boehringer et al. 1994) and the density of IGM is $\rho=10^{-27} \mathrm{~g} / \mathrm{cm}^{3}$ (Schindler et al. 1999). The GOLDMine database (Gavazzi et al. 2003) reports an infrared luminosity of $H_{\text {lum }}=8.70 \times 10^{8} L_{\odot}$ for VCC 1217 and a color $N U V-H=3.02 \mathrm{mag}$. With these characteristics VCC 1217 lies in the blue sequence of the Virgo cluster (see Fig. 3).

VCC 1217 consists of a low surface brightness disk whose structure is contaminated by several foreground stars. The central brightest envelope, shaped as an elongated ellipse, contains an excess of light in the north eastern part of the object (Fig. 2a). This is the brightest region of the galaxy and we will center all our subsequent radial analysis there. A secondary peak is found more to the south-west, offset by 15 arcsec.

The surface brightness profile was evaluated (after the masking the four most luminous foreground stars) with a modified version of the ellipse task in IRAF, centering the ellipses on the brightest spot and fitting the profile with an exponential law and a scale length of 19.1 arcsec.

As revealed by both the color map $u-i$ (Fig. 2b) and the RGB image (Fig. 2a), the brightest spot has a blue color of $u-$ $i=1.5$ (see Fig. 4), the secondary peak in the south-west of the galaxy is redder $(U-i=1.87)$. We performed a radial color analysis, integrating the $u, g, i$, and $N U V$ images on 12 elliptical annuli with a major axis of 1 arcmin, an axis ratio of $1.5 \mathrm{~m}$ and an inclination of the major axis of 50 degrees clockwise.

Again we masked the 4 more luminous stars superposed on the disk. The color profiles (Fig. 4) show a gradient of increasing color index by 0.4 mag with increasing distance from the center to 1 arcmin, where the contamination by the blue blobs is null.

\subsection{Spectroscopy}

A spectrum of the galaxy has been published by Gavazzi et al. (2004), and it is publicly available through GOLDMine (Gavazzi et al. 2003). It was obtained at the ESO $3.6 \mathrm{~m}$ telescope in drift scan mode, i.e. with the slit sliding over the whole galaxy area, thus representing the mean spectral characteristics of the object. We smoothed the spectrum by $5 \AA$ (Fig. 5) and measured the equivalent widths of the Balmer lines, which are all stronger than $5 \AA$ in absorption (as reported in Table 3), with no emission lines. In particular, the $\mathrm{H} \delta$ line has an $E W=13.4 \AA$ (adopting the convention that positive $E W$ means absorption), i.e. stronger than the threshold in the diagnostic diagrams of $\mathrm{k}+\mathrm{a}$ galaxies (e.g. Poggianti et al. 2004; Dressler et al. 1999). k+a galaxies are interpreted to be post star-burst (PSB) galaxies that underwent a sudden truncation of the star formation in the past $0.5-1.5 \mathrm{Gyr}$ (Couch \& Sharples 1987).

\section{The fireballs}

A complex of faint blue knots and filaments extends from the galaxy in south-east direction, up to $3.5 \mathrm{arcmin}(17 \mathrm{Kpc})$. They are outstanding in both the GALEX data (Fig. 2d) and in the RGB image prepared with the $u, g, i$ LBT images (Fig. 1, where the galaxy is saturated). We identify the brightest and clumpiest structures as "knots" (enumerating them from K-1 to K-8 east to west). Other structures with a lower surface brightness and visually more diffuse are labeled as "filaments", from F-1 to F-4.

Regions (highlighted in Figs. 1 and 2a,c) were selected on the basis of their detection on the GALEX images (with a resolution $>5$ arcsec), even though the LBT images offer a better resolution. This choice is dictated by our wanting to obtain the set of photometric measurements over the full spectral range for each feature, from UV to $i$-band, necessary for an SED fitting 


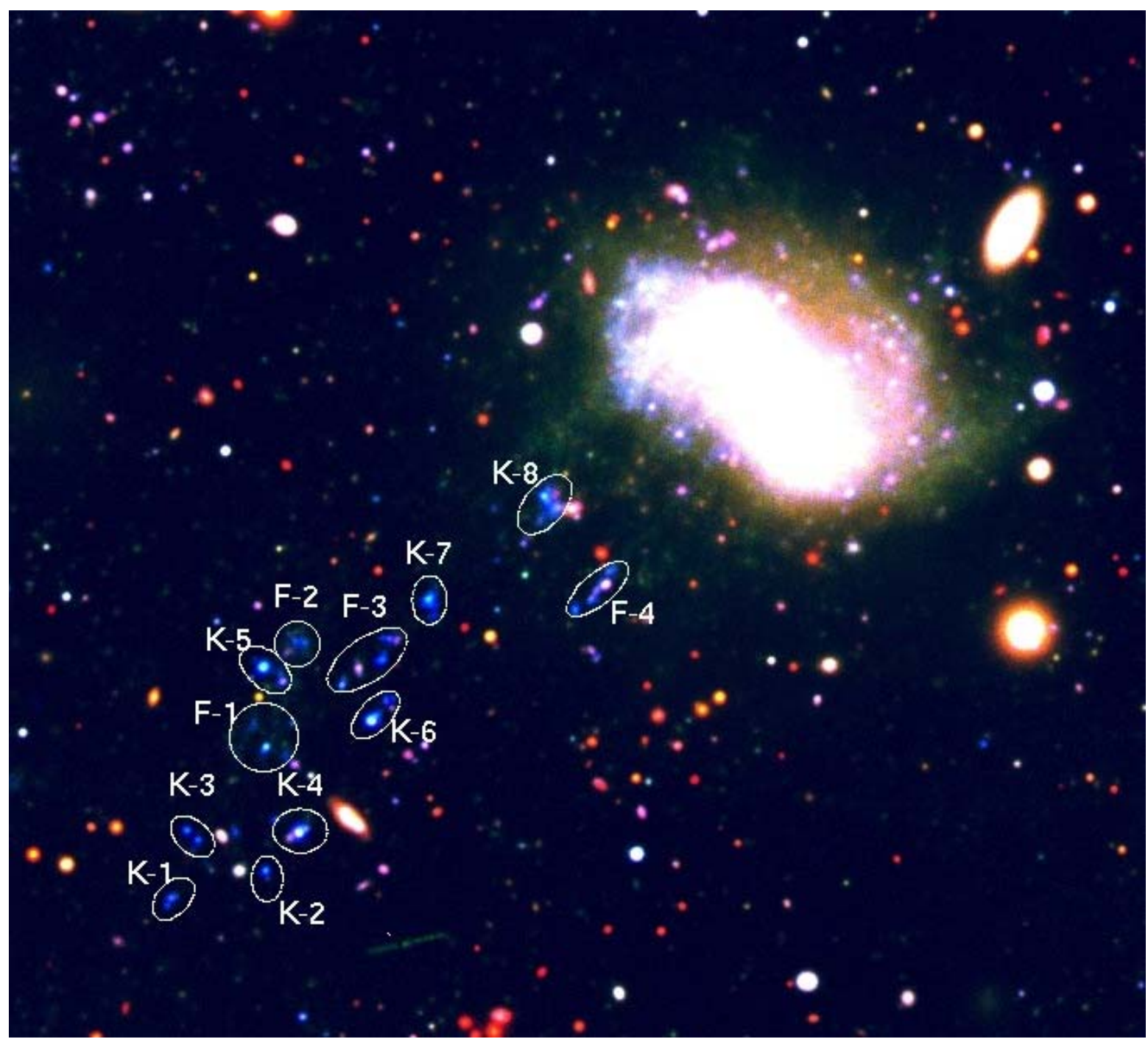

Fig. 1. High contrast RGB picture of VCC 1217 obtained from the LBT images ( $U$-spec, $g$-SDSS, $i$-SDSS), highlighting the morphology and the color of the blobs.

analysis (see Sect. 5), For instance, the region K-5 appears to be a bright knot in the $N U V$ image (Fig. 2d), while it is resolved into two distinct blobs by the LBT. The same holds for filaments (see for instance F-3), which might consist of fainter knots connected by diffuse regions. Although our regions do not exactly coincide with the ones in Hester et al. $(2010)^{2}$, the photometry in the $F U V$ and $N U V$ bands is generally consistent.

Figure 6 shows the fireballs in the two color differences $F U V-N U V$ and $u-g$. The filaments appear marginally redder than the knots with a mean color $\langle F U V-N U V\rangle_{F}=0.21$ and $\langle u-g\rangle_{F}=0.31$, while knots have $\langle F U V-N U V\rangle_{K}=-0.21$ and $\langle u-g\rangle_{K}=-0.05$. Only one knot is as red as the filaments (K-6 in $F U V-N U V, \mathrm{~K}-8$ in $u-g$ ). All the structures in the wake are much bluer than the galaxy $(F U V-N U V=0.66$ and $u-g=1.4)$. Moreover, there is a slight dependence of the color of knots/filaments on the distance from the galaxy; i.e., the ones located farther away are $\approx 0.5$ mag bluer than the closest ones.

\footnotetext{
2 The rationale for the small discrepancy between our regions and the ones in Hester et al. (2010), beside nomenclature, is twofold: 1. our analysis initiated before the appearance of Hester et al. (2010); 2. we positioned the regions on the basis of the $N U V$ detection, but the fine tuning and the division into knots and filaments was aided by our highresolution LBT image.
}

Various blobs display an $\mathrm{H} \alpha$ emission, as shown in Fig. 2c and in Table 4, with luminosities ranging from $8 \times 10^{36} \mathrm{erg} \mathrm{s}^{-1}$ (K-1) to $8 \times 10^{37} \mathrm{erg} \mathrm{s}^{-1}(\mathrm{~K}-6)$, consistently with the faint end of the HII luminosity function (Kennicutt et al. 1989). The signal-to-noise ratio of the image (see Sect. 2) is such that every star-forming region with a flux larger than $2.51 \times$ $10^{-16} \mathrm{erg} \mathrm{s}^{-1} \mathrm{~cm}^{-2} \mathrm{str}^{-1}$ can be detected (integrating $2 \sigma$ counts of the sky on the typical dimension of a blob, $10 \operatorname{arcsec}^{2}$ ). The $\mathrm{H} \alpha$ emission is concentrated in the knots farther than $2.3 \mathrm{Kpc}$ from the galaxy and only in one filament (F-3), which was said to consist of smaller knots. Consistently with the case of RB199 (Yoshida et al. 2008), the star formation resides in the most compact regions in the wake. According to Kennicutt (1998), the ongoing SFR is evaluated to be on the order of $10^{-3 /-4} M_{\odot} / \mathrm{yr}$ in each blob (see Table 4), with a cumulative SF in the entire wake of $\approx 1.9 \times 10^{-3} M_{\odot} / \mathrm{yr}$.

\section{SED fitting}

In order to reconstruct the star formation history of the blobs and of the galaxy, we generated spectral evolution models with the PEGASE2.0 code (Fioc \& Rocca-Volmerange 1997) and performed an SED-fitting using GOSSIP (Franzetti et al. 2008). For 

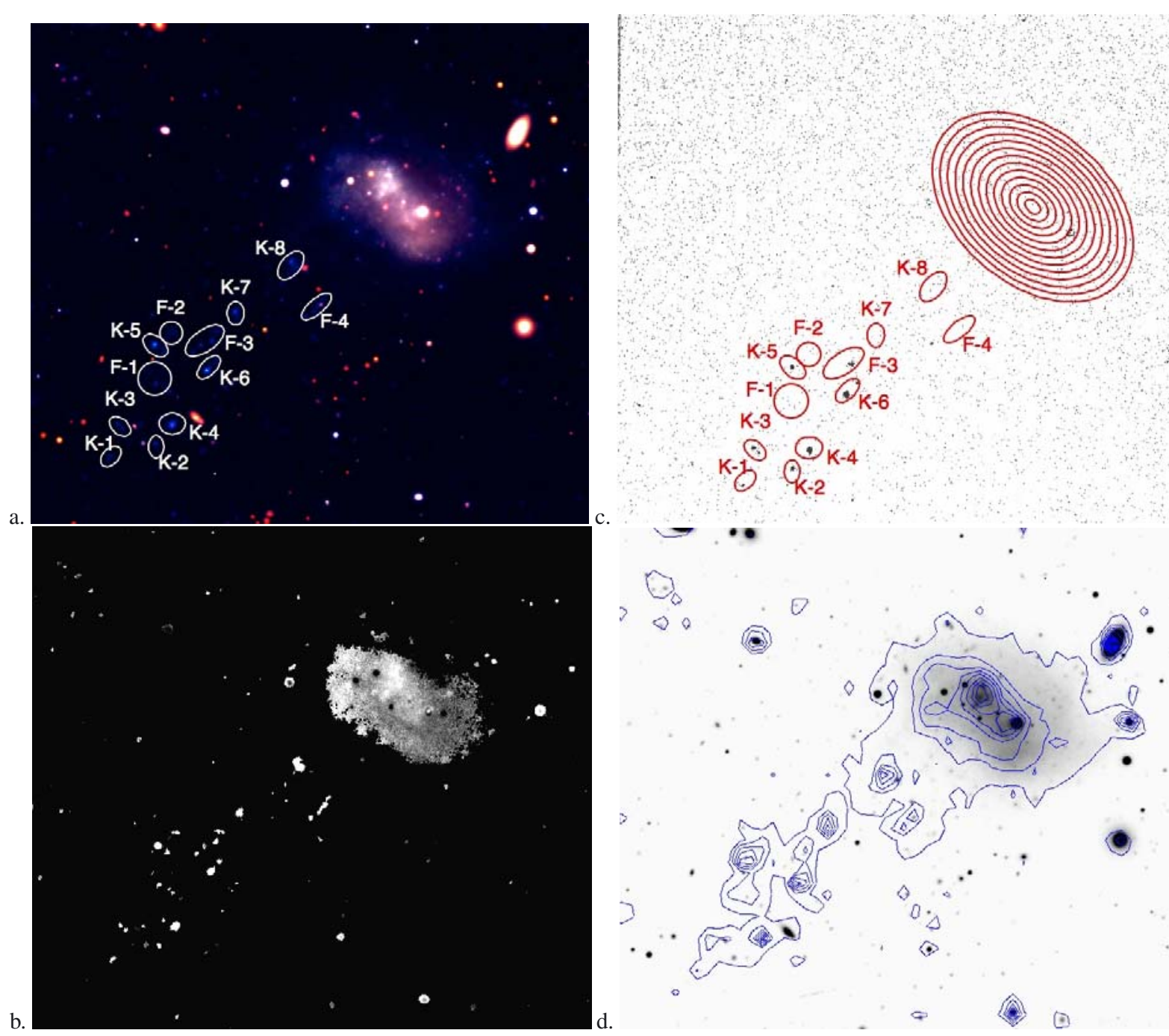

Fig. 2. a) Low-contrast RGB picture highlighting the galaxy structure and colors. b) $u-i$ image of the galaxy obtained from the ratio of the $u$ and $i$ images, each thresholded above $2 \sigma$ of the sky (outer black regions). In regions above the threshold the grey scale goes from white $(u-i=1.5)$ to black $(u-i=1.9)$ with increasing $u-i$ index. c) Superposed on the $\mathrm{H} \alpha$ image, the regions on which the photometry of the individual blobs has been evaluated and the concentric elliptical rings used to obtain the color profile of the galaxy (see Fig. 4) d) $N U V$ contours superposed on a low-contrast $g$ image.

Table 2. Photometry of VCC 1217 and its associated blobs.

\begin{tabular}{|c|c|c|c|c|c|c|c|}
\hline (1) & $\begin{array}{c}\text { Distance } \\
\text { (arcmin) } \\
\text { (2) }\end{array}$ & $\begin{array}{c}u \\
(\mathrm{mag}) \\
(3)\end{array}$ & $\begin{array}{c}g \\
(\mathrm{mag}) \\
(4)\end{array}$ & $\begin{array}{c}r \\
(\mathrm{mag}) \\
(5)\end{array}$ & $\begin{array}{c}i \\
(\mathrm{mag}) \\
(6)\end{array}$ & $\begin{array}{c}N U V \\
\text { (mag) } \\
(7)\end{array}$ & $\begin{array}{c}F U V \\
(\mathrm{mag}) \\
(8)\end{array}$ \\
\hline GAL & 0 & $15.98 \pm 0.03$ & $14.74 \pm 0.02$ & $14.39 \pm 0.02$ & $14.19 \pm 0.02$ & $17.36 \pm 0.022$ & $18.14 \pm 0.06$ \\
\hline K8 & 1.13 & $20.79 \pm 0.12$ & $20.42 \pm 0.03$ & $20.41 \pm 0.04$ & $20.16 \pm 0.05$ & $21.05 \pm 0.11$ & $20.92 \pm 0.19$ \\
\hline F4 & 1.28 & $21.36 \pm 0.16$ & $21.20 \pm 0.04$ & $21.17 \pm 0.05$ & $21.01 \pm 0.08$ & $21.63 \pm 0.15$ & $22.03 \pm 0.29$ \\
\hline K7 & 1.83 & $21.35 \pm 0.16$ & $21.31 \pm 0.04$ & $21.71 \pm 0.06$ & $21.98 \pm 0.11$ & $21.39 \pm 0.13$ & $21.27 \pm 0.21$ \\
\hline F3 & 2.14 & $20.92 \pm 0.13$ & $20.76 \pm 0.04$ & $20.49 \pm 0.04$ & $20.43 \pm 0.06$ & $21.52 \pm 0.14$ & $21.86 \pm 0.27$ \\
\hline K6 & 2.39 & $20.83 \pm 0.13$ & $20.94 \pm 0.04$ & $20.74 \pm 0.04$ & $20.96 \pm 0.07$ & $21.23 \pm 0.12$ & $21.28 \pm 0.22$ \\
\hline $\mathrm{F} 2$ & 2.40 & $21.62 \pm 0.18$ & $21.04 \pm 0.04$ & $21.07 \pm 0.05$ & $20.91 \pm 0.07$ & $21.75 \pm 0.15$ & $21.69 \pm 0.26$ \\
\hline K5 & 2.55 & $20.84 \pm 0.13$ & $20.78 \pm 0.04$ & $20.82 \pm 0.04$ & $20.90 \pm 0.07$ & $20.90 \pm 0.11$ & $20.80 \pm 0.18$ \\
\hline F1 & 2.74 & $21.00 \pm 0.14$ & $20.58 \pm 0.03$ & $20.67 \pm 0.04$ & $20.65 \pm 0.07$ & $21.25 \pm 0.12$ & $21.41 \pm 0.23$ \\
\hline K4 & 3.00 & $20.94 \pm 0.13$ & $21.04 \pm 0.04$ & $20.64 \pm 0.04$ & $20.65 \pm 0.06$ & $21.31 \pm 0.13$ & $21.17 \pm 0.21$ \\
\hline K2 & 3.16 & $22.51 \pm 0.26$ & $22.83 \pm 0.08$ & $22.67 \pm 0.09$ & $23.22 \pm 0.20$ & $23.13 \pm 0.27$ & $22.92 \pm 0.42$ \\
\hline K3 & 3.30 & $21.93 \pm 0.20$ & $22.21 \pm 0.06$ & $22.03 \pm 0.07$ & $22.43 \pm 0.14$ & $22.19 \pm 0.19$ & $21.84 \pm 0.27$ \\
\hline K1 & 3.55 & $22.41 \pm 0.25$ & $22.44 \pm 0.07$ & $22.45 \pm 0.08$ & $22.6 \pm 0.15$ & $23.01 \pm 0.26$ & $22.37 \pm 0.34$ \\
\hline
\end{tabular}

Notes. (1) GAL = VCC 1217, K-\# Knots, F-\# Filaments. (2) Projected distance from the center of VCC 1217. (3) to (8) The photometric uncertainties are the quadratic sum of the ZP error and Poisson error. 


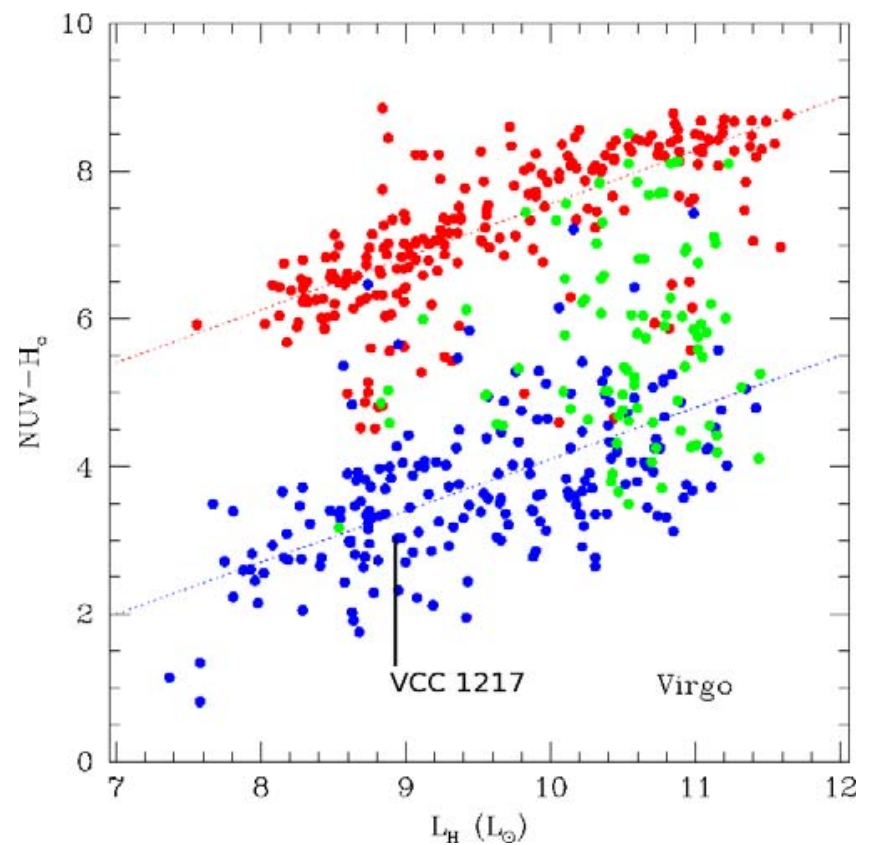

Fig. 3. $N U V$-H color luminosity diagram of the Virgo cluster (from the GOLDMine database, Gavazzi et al. 2003). Objects are color-coded according to morphological classification: red for early type galaxies (elliptical and S0), blue for disk dominated late type galaxies ( $\mathrm{Sc}$ to Sd), green for disk+bulge galaxies ( $\mathrm{Sa}$ to $\mathrm{Sb}$ ). Dashed lines represent the best fit to the red and blue sequences. The position of VCC 1217 is highlighted.

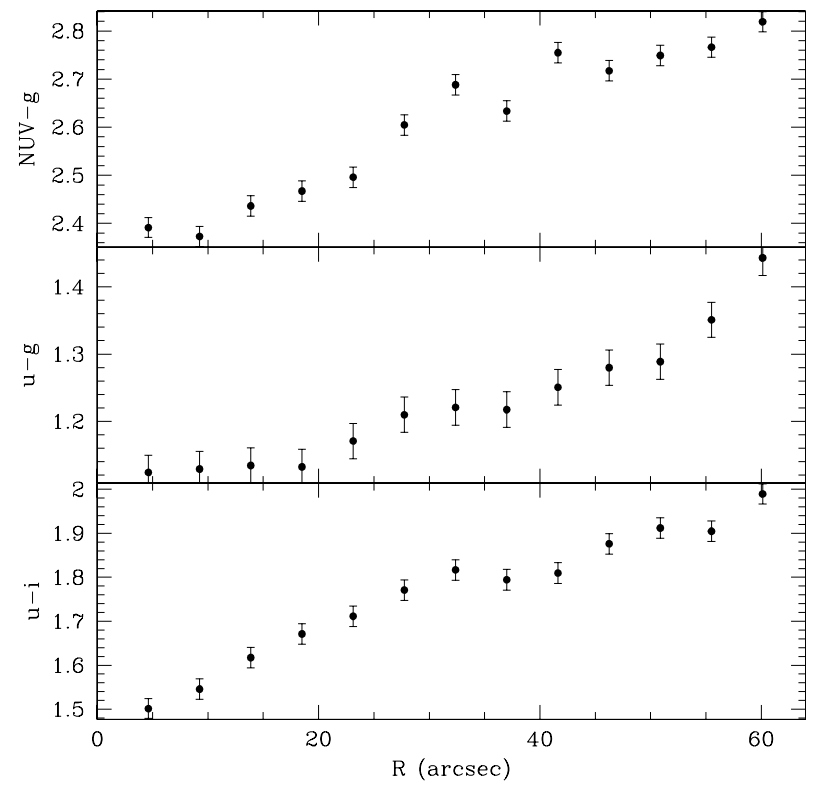

Fig. 4. $N U V-g$ (top), $u-g$ (center), $u-i$ (bottom) color profile of VCC 1217, obtained in the concentric annuli of Fig. 2c.

both the galaxy and the filaments/knots, which are analyzed separately, the procedure consists in

- giving one or more star formation histories, i.e. $\operatorname{SFR}(t)$, as an input to PEGASE;

- generating synthetic spectra at fixed times;

- running the SED-fitting in GOSSIP between the synthetic spectra and the photometric points of the objects.

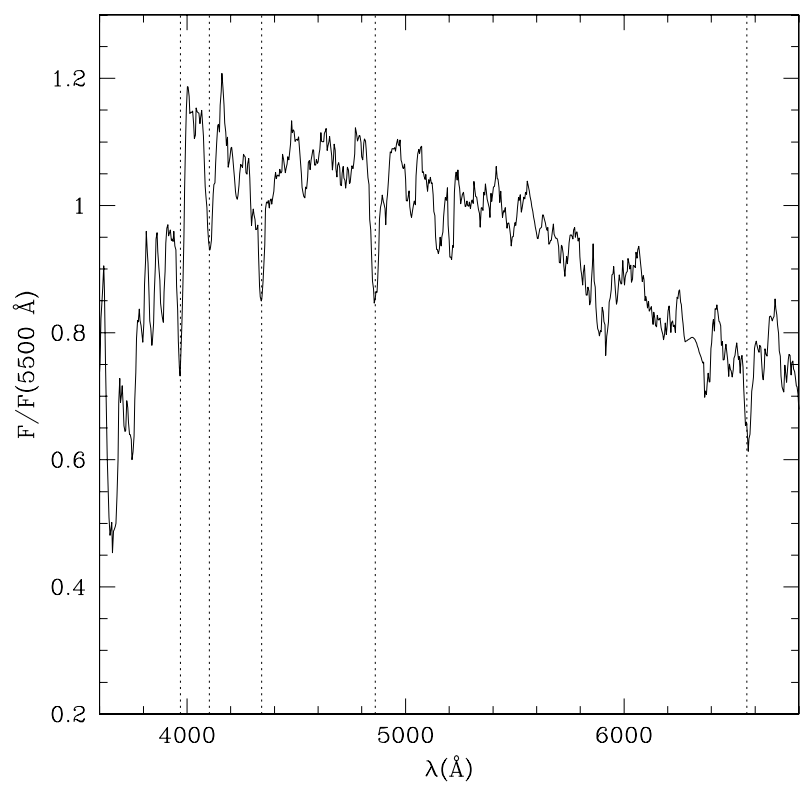

Fig. 5. Spectrum of VCC 1217 obtained in drift scan mode at the ESO $3.6 \mathrm{~m}$ telescope. The flux is normalized to $F(5500 \AA)$. Dashed lines highlight the position of the Balmer series.
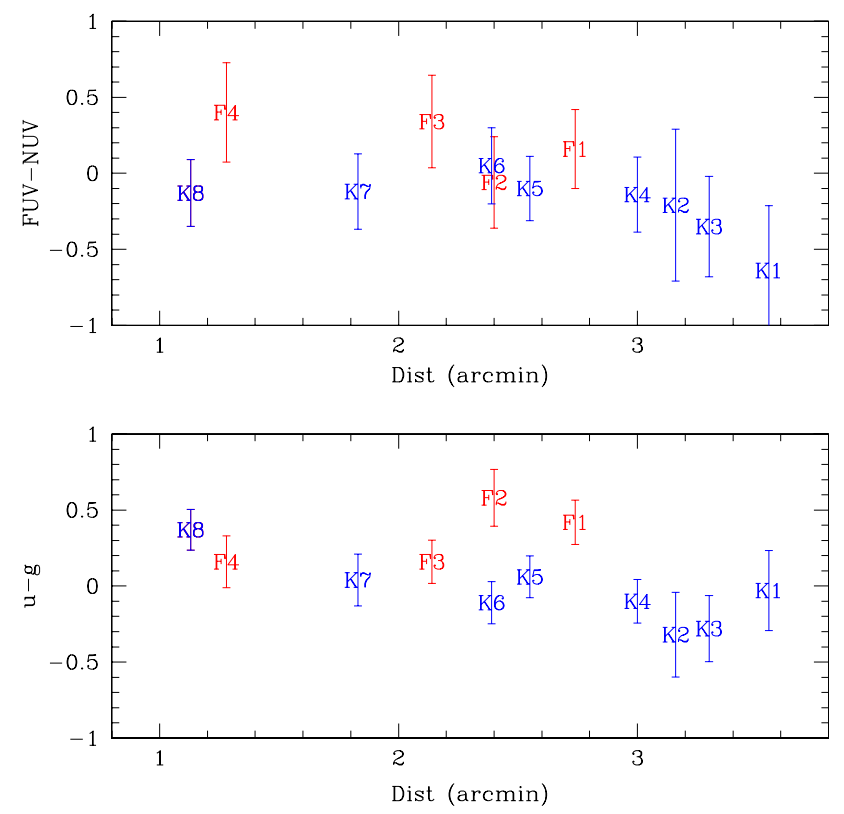

Fig. 6. Colors of the knots (blue) and filaments (red).

\subsection{The galaxy}

We model the spectral evolution of the galaxy assuming a Salpeter IMF with an upper mass of $120 M_{\odot}$, a null initial metallicity, and a star formation history "a la Sandage" (as reported in Gavazzi et al. 2003), with a discrete set of $\tau$ parameters. Since we want to test the hypothesis of ram pressure stripping on the galaxy, in this phase we simulate the effect of the gas depletion including in the models a truncation of the star formation at a given characteristic time (Truncation time, $t_{\text {trunc }}$ ). The star formation history becomes

$S F R(t)=\left\{\begin{aligned} \frac{t}{\tau^{2}} \mathrm{e}^{-\frac{t^{2}}{\tau^{2}}} & \text { if } t<t_{\text {trunc }} \\ 0 & \text { if } t>t_{\text {trunc }}\end{aligned}\right.$ 


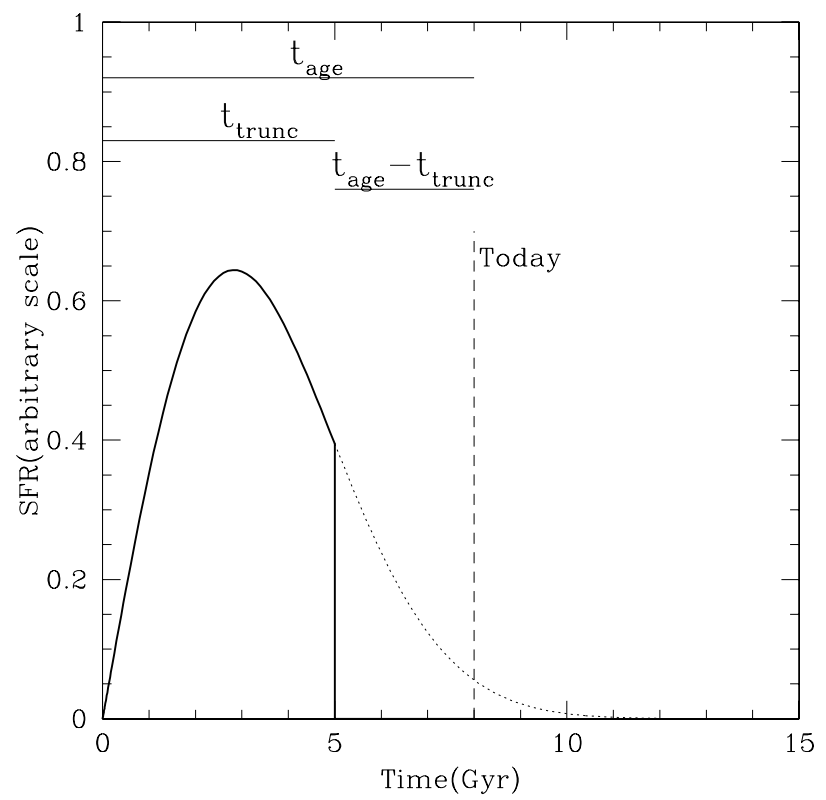

Fig. 7. A sample star formation history from the library, with $\tau=4 \mathrm{Gyr}$, $t_{\mathrm{age}}=8 \mathrm{Gyr}$ and $t_{\text {trunc }}=5 \mathrm{Gyr}$.

In Fig. 7 we show a sample star formation history in the models library and highlight the different timescales referred to in the article: $t_{\text {age }}$ is the time from the onset of star formation to now, $t_{\text {trunc }}$ the time from the formation until the end of star formation activity, and $t_{\text {age }}-t_{\text {trunc }}$ the period between the previous two. The grid of parameters was built with $\tau$ ranging from 1 to 20 Gyr with 1 Gyr step and $t_{\text {trunc }}$ from 1 to 13 Gyr with $1 \mathrm{Gyr}$ step, while $t_{\text {age }}$ spans from 0 to 13.5 Gyr with a step of $100 \mathrm{Myr}$, for a total of $35 \mathrm{~K}$ spectra. We notice that in the model building we didn't include any fixed age for the start of star formation activity. or any correction for dust extinction, since it is negligible for low-mass galaxies (see Fig. 8 of Cortese et al. 2008 and 3-4 of Lee et al. 2009). We ran GOSSIP and evaluated the parameters and their probability distribution functions (PDFs). We find that the $t_{\text {age }}$, the $t_{\text {trunc }}$, and $\tau$ are not well costrained. However, we computed the probability distribution function of $t_{\text {age }}-t_{\text {trunc }}$, representing the lookback time at which the truncation of star formation occurred, and this parameter results very well constrained in $t_{\text {age }}-t_{\text {trunc }}=200_{-90}^{+90}$ Myr. A halting of the star formation approximately $200 \mathrm{Myr}$ ago is in accordance with the PSB signature in the galaxy spectrum (the PDF is given in Fig. 8). The stellar mass of the galaxy evaluated from the normalization to the fit $\left(M_{\text {star }}=3 \times 10^{8} M_{\odot}\right)$ turns out to be in fair agreement with the stellar mass evaluation from the optical data (Bell et al. 2007), $M_{\text {star }}=3.8 \times 10^{8} M_{\odot}$.

\subsection{The fireballs}

For the fireballs we computed a sample of synthetic spectra with PEGASE2.0 assuming a Salpeter IMF, a subsolar initial metallicity, and the following set of star formation history models:

- single burst

$\operatorname{SFR}(t)=\delta(0)$

- constant star formation rate

$\operatorname{SFR}(t)=\operatorname{SFR} R_{0}$

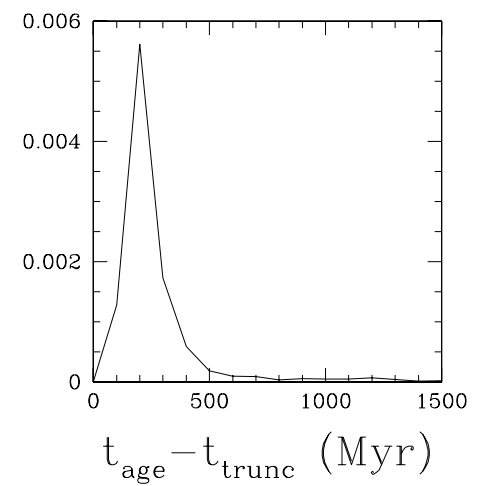

Fig. 8. Probability distribution functions of the $t_{\text {age }}-t_{\text {trunc }}$ parameter for VCC 1217.

Table 3. Spectroscopy of VCC 1217.

\begin{tabular}{cccc}
\hline \hline & $\begin{array}{c}\text { Wavelength } \\
\AA\end{array}$ & $\begin{array}{c}\text { Continuum } \\
F / F(5500 \AA)\end{array}$ & $E W$ \\
\hline $\mathrm{H} \epsilon$ & 3969.1 & 1.02 & 8.0 \\
$\mathrm{H} \delta$ & 4109.7 & 1.18 & 13.4 \\
$\mathrm{H} \gamma$ & 4339.8 & 1.06 & 7.4 \\
$\mathrm{H} \beta$ & 4861.0 & 1.07 & 9.0 \\
$\mathrm{H} \alpha$ & 6568.3 & 0.78 & 6.2 \\
\hline
\end{tabular}

- exponential decrement of SFR rate

$\operatorname{SFR}(t)=S F R_{0} \exp (-t / \tau)$

with a finite set of $\tau$ parameters $(\tau=10,20,40,60,80,100$, 200, 300, 400, 500, 600, 700, 800, 900, 1000, 1200, 1400, 1600, 1800, $2000 \mathrm{Myr})$.

Again we ran the SED fitting with GOSSIP, and extracted the best fits (Table 4) and evaluated their PDFs. The stellar masses of the fireballs (derived from the normalization to the SED) range from $3.9 \times 10^{4}$ to $5.0 \times 10^{5} M_{\odot}$, which are typical dimensions of a giant molecular cloud / HII region (Kennicutt et al. 1989). For such small objects we neglected the internal absorption.

We want to stress that we prefer not to improve the quality of the fit by replacing of our star formation history models (with at most one free parameter) with additional ad-hoc bursts.

Table 4 contains the parameters of the SEDs. Most of the knots SEDs are described well by a simple exponential star formation history with age $t<400 \mathrm{Myr}$ (excluding K4). We refer to age as the time from the ignition of the star formation to now. Filaments appear to be all together older than blobs $\left(\langle A g e\rangle_{\mathrm{F}}=850 \mathrm{Myr}\right)$ with star formation activity stretched over a longer period of time.

While the age parameter is well constrained, as shown by the spiky shape of the PDFs in Fig. 9 (left panels), not much can be said about $\tau$, generally just a lower limit. From the PDFs of $\tau$ we can also conclude that a single burst model (which corresponds to an exponential model with $\tau \rightarrow 0$ ) can be ruled out, while a model with a constant star formation rate (which corresponds to an exponential model with $\tau \rightarrow \infty$ ) is consistent with the data, especially for the young knots. That $\tau$ is longer for the knots than the age indicates that star formation activity is still going on, in accordance with the $\mathrm{H} \alpha$ emission in most of the knots.

For such young objects it is impossible to constrain the star formation history more precisely by simply letting $\tau$ vary. This is illustrated in a color-color diagram (Fig. 10) where evolutionary tracks obtained with various $\tau$ are plotted with the data. At young 
Table 4. Analysis of the blobs.

\begin{tabular}{cccccc}
\hline \hline Name & $\begin{array}{c}\text { Distance from } \\
\text { the galaxy }(\mathrm{arcmin})\end{array}$ & $\begin{array}{c}\mathrm{H} \alpha \text { Flux } \\
\left(10^{-16} \mathrm{erg} / \mathrm{s} / \mathrm{cm}^{2}\right)\end{array}$ & $\begin{array}{c}\text { SFR, Kennicutt law } \\
\left(10^{-3} M_{\odot} / \mathrm{yr}\right)\end{array}$ & $\begin{array}{c}\text { Mass } \\
\left(10^{5} M_{\odot}\right)\end{array}$ & $\begin{array}{c}\text { Age } \\
(\mathrm{Myr})\end{array}$ \\
\hline K8 & 1.13 & - & - & 3.74 & $130_{-150}^{+454}$ \\
F4 & 1.28 & - & - & 2.45 & $620_{-90}^{+160}$ \\
K7 & 1.83 & - & - & 0.93 & $80_{-23}^{+23}$ \\
F3 & 2.14 & 7.76 & 0.21 & 5.49 & $1400_{-150}^{+350}$ \\
K6 & 2.39 & 0.25 & 0.69 & 2.03 & $390_{-87}^{+87}$ \\
F2 & 2.40 & - & - & 2.20 & $780_{-186}^{+192}$ \\
K5 & 2.55 & 6.92 & 0.19 & 1.18 & $170_{-39}^{+39}$ \\
F1 & 2.74 & - & & 3.48 & $640_{-144}^{+248}$ \\
K4 & 3.00 & 0.16 & 0.10 & 3.79 & $740_{-122}^{+122}$ \\
K2 & 3.16 & 6.22 & 0.16 & 0.35 & $330_{-123}^{+123}$ \\
K3 & 3.30 & 8.31 & 0.23 & 0.55 & $140_{-52}^{+52}$ \\
K1 & 3.55 & 2.34 & 0.06 & 0.40 & $260_{-146}^{+146}$ \\
\hline
\end{tabular}
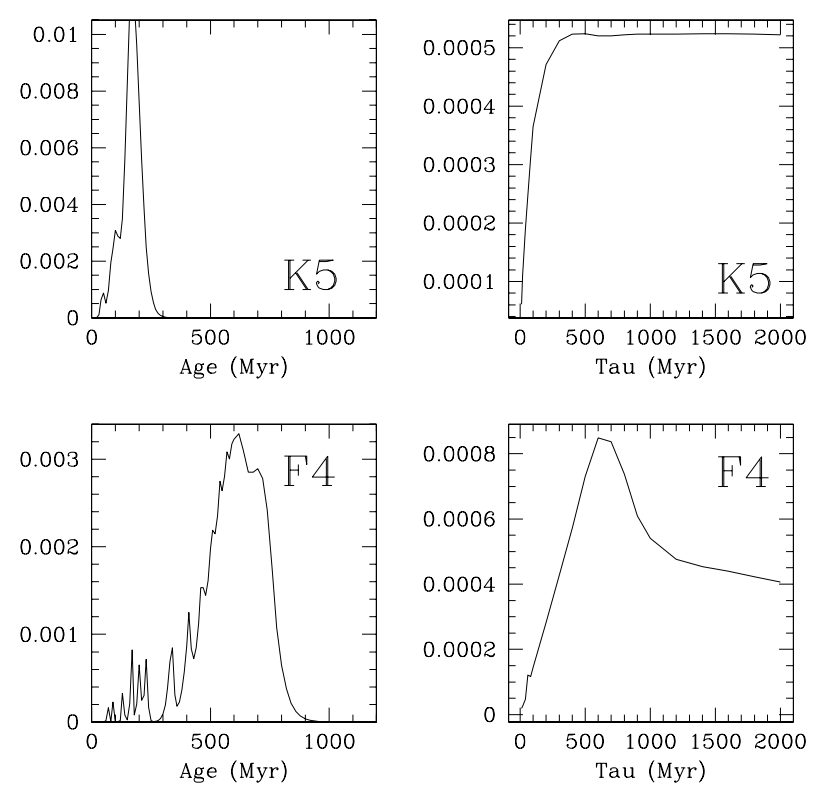

Fig. 9. Probability distribution functions of the Age and $\tau$ parameters for a sample knot and a sample blob.

ages different tracks are indistinguishable until the stellar population is more evolved (compare with Figs. 13-14 of Yoshida et al. 2008: the fireballs associated with RB199 are older than the ones in the present study). Figure 11 collects some SEDs of the blobs and the galaxy, showing the quality of the fits. The features in the wake of VCC 1217 all look very similar and extremely different from the galaxy.

\section{Ram pressure stripping}

Adopting the classical Gunn \& Gott (1972) criterion for ram pressure

$\rho_{\mathrm{IGM}} v^{2} \geq 2 \pi G \Sigma_{\text {star }} \Sigma_{\text {gas }}$,

where $\rho_{\mathrm{IGM}}$ is the intracluster density, $v$ the infall velocity, and $\Sigma_{\text {star,gas }}$ the density of the star and gas components in the galaxy,

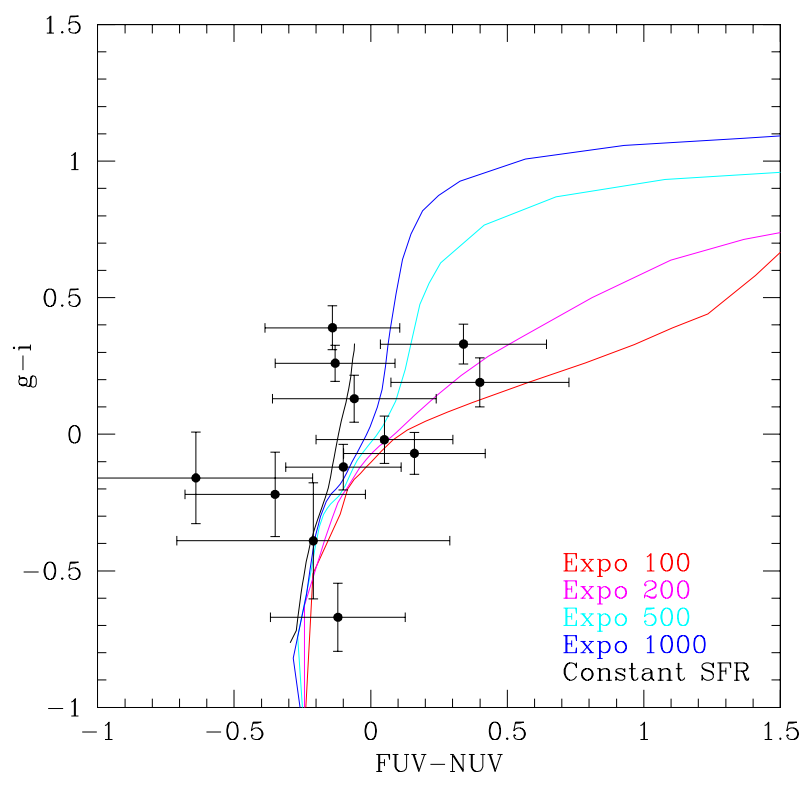

Fig. 10. Color-color diagram of $F U V-N U V$ and $g-i$ for the blobs in the wake of VCC 1217. The solid lines are the predictions of some star formation history models: constant star formation rate and exponential star formation rate with $\tau$ 100, 200, 500 and $1000 \mathrm{Myr}$, color coded as in the legend.

and assuming an exponential profile for the stellar and gas component, the radius at which ram pressure becomes efficient can be estimated as (Domainko et al. 2006)

$R_{\text {strip }}=0.5 R_{0} \ln \left(\frac{G M_{\text {star }} M_{\text {gas }}}{v^{2} \rho_{\mathrm{IGM}} 2 \pi R_{0}^{4}}\right)$,

and the stripped mass as

$M_{\text {strip }}=M_{\text {gas }}\left(\frac{R_{\text {strip }}}{R_{0}}+1\right) \exp \left(-\frac{R_{\text {strip }}}{R_{0}}\right)$.

Adopting $M_{\text {star }}=3.8 \times 10^{8} M_{\odot}, M_{\text {star }} / M_{\text {gas }} \approx 1$ (for late type galaxies, e.g. Boselli 2002), $\rho=6 \times 10^{-4} \mathrm{~cm}^{-3}$ for the IGM density of the Virgo cluster at the projected distance of $0.3 \mathrm{Mpc}$ from 


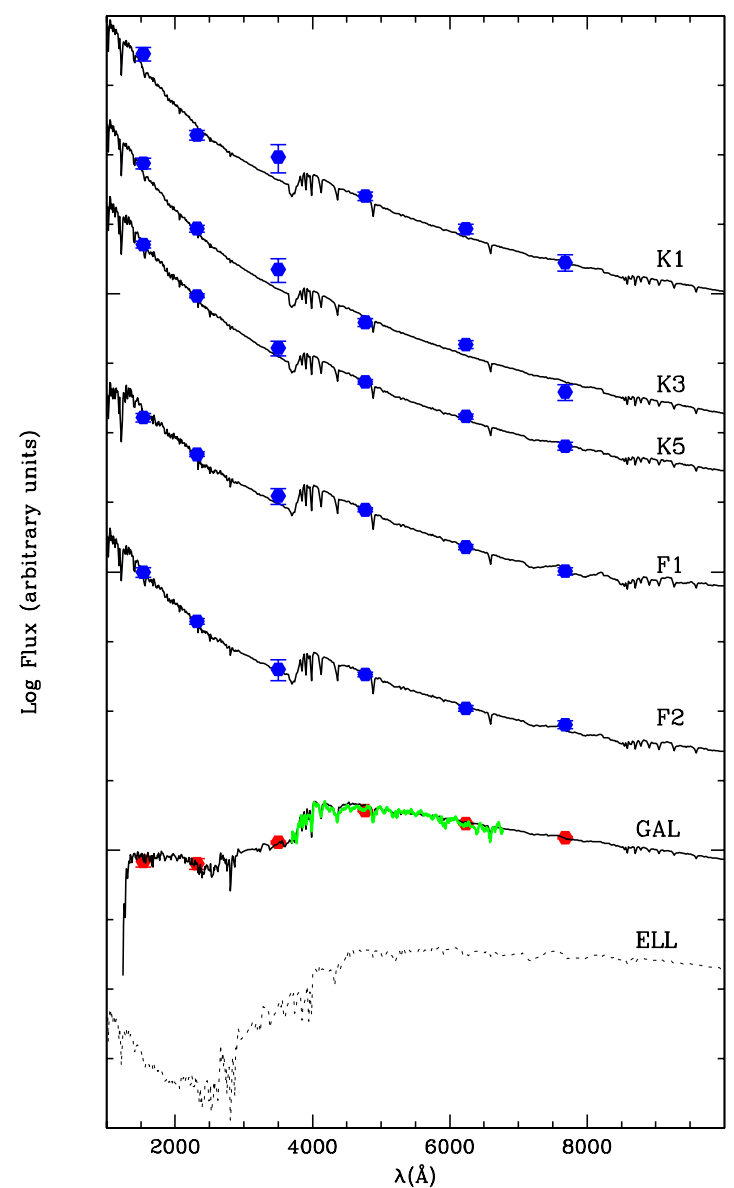

Fig. 11. Photometric points for the blobs (blue) and their best fit SEDs. Photometric points (red), observed optical spectrum (green) for the galaxy VCC 1217, and best-fit model with a truncated Sandage star formation history. The dashed line represents the spectrum of an old quiescent elliptical galaxy for comparison with a Sandage star formation history with parameter age $=13 \mathrm{Gyr}$ and $\tau=4 \mathrm{Gyr}$.

M 87 (Schindler et al. 1999), an infall velocity of $1000 \mathrm{~km} \mathrm{~s}^{-1}$, and the typical scale length computed in Sect. 3.1, We find that VCC 1217 is unable to retain its gas at any radius if subjected to ram pressure stripping $\left(R_{\text {strip }}=0.0 \mathrm{Kpc}\right)$, leading to totally depleted of gas $\left(M_{\text {strip }}=M_{\text {gas }}\right)$.

\section{Discussion}

Although when looking just at UV data ${ }^{3}$ we cannot rule out the system of blobs being the remnant of a dwarf irregular galaxy in some stage of merging with VCC 1217, the LBT data resolves the tail feature into separated compact (at most filamentary) blobs, revealing that the morphology of the system is not consistent with the merging scenario. Several aspects of our analysis indicate that the ram pressure stripping picture is the most favorable one (in agreement with Hester et al. 2010), suggesting that VCC 1217 has been recently stripped by the interaction with the Virgo cluster IGM. The color profiles, the spectroscopy, and SED fitting of the galaxy, all support the scenario consisting in a truncation of the star formation in the last few hundred Myr.

The ensemble of blue knots and filaments stretching more than $17 \mathrm{Kpc}$ south east of the galaxy is a remarkable feature.

\footnotetext{
3 See http://www.galex.caltech.edu/media/images/ glx2010-02f_img01.jpg
}

Their SEDs are consistent with very young stellar objects, born in the last few hundred Myr, consistent with the timing of the gas depletion from the galaxy.

Similar objects have been observed in other clusters, but the phenomenon appears to be rare. Cortese et al. (2007) report the discovery of two complexes of stellar tails and blue bright blobs associated with two spiral galaxies infalling in massive clusters at $z \approx 0.2$. Yoshida et al. (2008) have analyzed a complex of $\mathrm{H} \alpha$ emitting fireballs extending from the Coma cluster galaxy RB199, up to $80 \mathrm{Kpc}$. Like VCC 1217, RB199 and 131124012040 in Abell 1689 have $\mathrm{k}+\mathrm{a}$ spectra, while 235144-260358 in Abell 2667 is still a star-forming galaxy. There is however a different scale(NON CAPISCO) for the objects in this study and the ones in the literature, both for the galaxies and the blobs. RB199 is estimated to have a mass of $3-4 \times 10^{9} M_{\odot}$, i.e. 10 times more massive than the one of VCC 1217. Yoshida et al. compute a typical mass of the fireballs associated to RB199 of $\approx 10^{7-8} M_{\odot}$ and a total $L_{\mathrm{H} \alpha}=2 \times 10^{39} \mathrm{erg} \mathrm{s}^{-1}$, while the blobs in the present study range from $10^{4.6}$ to $10^{5.7} M_{\odot}$ in mass and have a total $\mathrm{H} \alpha$ luminosity of $2 \times 10^{38} \mathrm{erg} \mathrm{s}^{-1}$. Also the computed ages of the blobs are different, 500-1000 Myr for the complex stretching from RB199 and less than 400 Myr for most of the ones in the complex southwest of VCC 1217.

The complex in the present study appears to be a scaled down version of the one in Yoshida et al. (2008) because all its characteristic dimensions (galaxy mass, blobs masses, $\mathrm{H} \alpha$ luminosities) are approximately 10 times smaller than the ones in RB199.

Besides the limited number statistics, this occurrence might arise because the wake associated to VCC 1217 would be too faint to be seen at the distance of Coma, but also because in a lower density environment, such as Virgo, low-mass galaxies are primarily affected by ram pressure (Bekki 2009).

We note that this kind of event is extremely rare in the Universe, but the phenomenon happens on a variety of mass scales. Different simulations (e.g. Tommesen et al. 2010; Kapferer et al. 2009) have studied the impact of ram pressure in the distribution of gas in a galaxy, producing mock observations in $\mathrm{HI}, \mathrm{H} \alpha$ (and X-rays) that are similar to the observed tails. Various mechanisms are proposed for the $\mathrm{H} \alpha$ emission in the wakes. Kenney et al. (2008) suggest that $\mathrm{H} \alpha$ emission in the wake can be caused by thermal conduction from the IGM and turbulent shock heating. In the current case, however, it is more likely to be associated with star formation, since the blobs are also seen optically and have the SEDs typical of young star complexes.

The simulations by Kapferer et al. (2009) conclude that turbulence in the wake can lead to gravitational instability and to the formation of stars up to $100 \mathrm{Kpc}$ behind the stripped galaxy. The observed pictures of VCC 1217 (Fig. 2 a,b,c) are remarkably similar to the mock observations (see Figs. 9-12 in Kapferer et al. 2009). We cannot directly compare the number of new stars formed in the wake since the simulations were run when assuming a stellar mass of $2 \times 10^{10} M_{\odot}$ and a dark matter halo of $10^{12} M_{\odot}$ for the test galaxy, while VCC 1217 is significantly smaller and the dependence on mass of the combined effect of ram pressure, turbulence and gravitational instability has to be investigated further.

We find that, after approximately $200 \mathrm{Myr}$ from the stripping event, the number of new stars formed in the wake is $\approx 2.5 \times$ $10^{6} M_{\odot}$ (i.e. the sum of the masses of the blobs, see Table 4), $1 / 100$ of the whole mass of the stripped galaxy. Not detecting HI (Hoffman et al. 1989) is not surprising: if assuming a residual gas mass similar to the mass of the blobs, it lies under the Arecibo detection limit. 


\section{Conclusion}

We propose that VCC 1217 just interacted for the first time with the Virgo cluster and undergone a sudden truncation of its gas content owing to ram pressure stripping. Turbulence in the wake can lead to gravitational instability and lead to collapse the densest parts of the wake, with the subsequent birth of stars. The analysis by Hester et al. (2010) is confirmed and reinforced by the spectroscopic inspection of the galaxy and more notably by deep LBT imaging.

It is already known that, in the first stage of the interaction with the IGM, a galaxy can form a tail of ionized gas stretching up to $>50 \mathrm{Kpc}$. Examples of $\mathrm{H} \alpha$ tails can be found in the Virgo cluster (NGC 4522, Kenney \& Koopmann 1999) and in Abell 1367 (97-079 and 97-073, Gavazzi et al. 2001; the BIG group, Cortese et al. 2006). A recent survey of the Coma cluster by Yagi et al. (2010) shows that almost all blue galaxies in the core of this cluster reveal tails and distorted $\mathrm{H} \alpha$ profiles when observed with a 10-m class telescope.

In spite of the high frequency of cometary $\mathrm{H} \alpha$ structures associated with IGM-interacting galaxies, the inset of star formation in the wakes is less common and not fully understood. For instance within the Yagi et al. (2010) sample, only two objects show signs of star formation along the trail (GMP3016 and RB199). VCC 1217 so far represents the closest and smallest known object with this unusual feature.

Acknowledgements. We thank Alessandro Boselli and Luca Cortese for the useful discussions and the LBT Survey Center (LBC) for carrying out the observation and for technical support during the reduction and analysis. This research has made use of the GOLDMine Database. We acknowledge the anonymous referee for constructive criticism.

\section{References}

Bekki, K. 2009, MNRAS, 399, 2221

Bell, E. F., McIntosh, D. H., Katz, N., \& Weinberg, M. D. 2003, ApJS, 149, 289 Böhringer, H., Briel, U. G., Schwarz, R. A., et al. 1994, Nature, 368, 828
Boselli, A. 2001, Astrophys. Space Sci. Suppl., 277, 40

Boselli, A., \& Gavazzi, G. 2006, PASP, 118, 517

Boselli, A., Boissier, S., Cortese, L., \& Gavazzi, G. 2008, ApJ, 674, 742

Couch, W. J., \& Sharples, R. M. 1987, MNRAS, 229, 423

Cortese, L., Gavazzi, G., Boselli, A., et al. 2006, A\&A, 453, 847

Cortese, L., Marcillac, D., Richard, J., et al. 2007, MNRAS, 376, 157

Cortese, L., Boselli, A., Franzetti, P., et al. 2008, MNRAS, 386, 1157

Domainko, W., Mair, M., Kapferer, W., et al. 2006, A\&A, 452, 795

Draine, B. T., \& Lee, H. M. 1984, ApJ, 285, 89

Dressler, A., Smail, I., Poggianti, B. M., et al. 1999, ApJS, 122, 51

Fioc, M., \& Rocca-Volmerange, B. 1997, A\&A, 326, 950

Franzetti, P., Scodeggio, M., Garilli, B., Fumana, M., \& Paioro, L. 2008, Astronomical Data Analysis Software and Systems XVII, 394, 642

Gavazzi, G., Pierini, D., \& Boselli, A. 1996, A\&A, 312, 397

Gavazzi, G., Boselli, A., Scodeggio, M., Pierini, D., \& Belsole, E. 1999 MNRAS, 304, 595

Gavazzi, G., Boselli, A., Mayer, L., et al. 2001, ApJ, 563, L23

Gavazzi, G., Boselli, A., Donati, A., Franzetti, P., \& Scodeggio, M. 2003, A\&A, 400,451

Gavazzi, G., Zaccardo, A., Sanvito, G., Boselli, A., \& Bonfanti, C. 2004, A\&A, 417, 499

Gavazzi, G., Boselli, A., Cortese, L., et al. 2006, A\&A, 446, 839

Gavazzi, G., Fumagalli, M., Cucciati, O., \& Boselli, A. 2010, A\&A, 517, A73

Gunn, J. E., \& Gott, J. R., III 1972, ApJ, 176, 1

Haines, C. P., Merluzzi, P., Mercurio, A., et al. 2006, MNRAS, 371, 55

Hester, J. A., Seibert, M., Neill, J. D., et al. 2010, ApJ, 716, L14

Hoffman, G. L., Williams, H. L., Salpeter, E. E., Sandage, A., \& Binggeli, B. 1989, ApJS, 71, 701

Kapferer, W., Sluka, C., Schindler, S., Ferrari, C., \& Ziegler, B. 2009, A\&A, 499, 87

Kenney, J. D. P., \& Koopmann, R. A. 1999, AJ, 117, 181

Kenney, J. D. P., Tal, T., Crowl, H. H., Feldmeier, J., \& Jacoby, G. H. 2008, ApJ, 687, L69

Kennicutt, R. C., Jr. 1998, ApJ, 498, 541

Kennicutt, R. C., Jr., Edgar, B. K., \& Hodge, P. W. 1989, ApJ, 337, 761

Lee, J. C., Gil de Paz, A., Tremonti, C., et al. 2009, ApJ, 706, 599

Nilson, P. 1973, Nova Acta Regiae Soc. Sci. Upsaliensis Ser. V, 0

Penny, S. J., \& Conselice, C. J. 2010, [arXiv: 1009. 2922]

Poggianti, B. M., Bridges, T. J., Komiyama, Y., et al. 2004, ApJ, 601, 197

Schindler, S., Binggeli, B., \& Boehringer, H. 1999, A\&A, 343, 420

Smith, R. J., Lucey, J. R., Hammer, D., et al. 2010, MNRAS, 408, 1417

Tonnesen, S., \& Bryan, G. L. 2010, ApJ, 709, 1203

Yagi, M., Yoshida, M., Komiyama, Y., et al. 2010, AJ, 140, 1814

Yoshida, M., Yagi, M., Komiyama, Y., et al. 2008, ApJ, 688, 918 